\title{
Research note: The ability of individuals with right-hemisphere damage to use context under conditions of focused and divided attention
}

\author{
Carol L. Leonard ${ }^{\mathrm{a}, *}$, Shari R. Baum ${ }^{\mathrm{b}}$ \\ ${ }^{a}$ Kunin-Lunenfeld Applied Research Unit, Baycrest Centre for Geriatric Care, \\ 3560 Bathurst Street, Toronto, Ont., Canada M6A 2E1 \\ ${ }^{\mathrm{b}}$ School of Communication Sciences and Disorders, McGill University, \\ 1266 Pine Avenue West, Montreal, Que., Canada H3G 1A8
}

Received 16 November 2004; received in revised form 30 March 2005; accepted 31 March 2005

\begin{abstract}
A word-monitoring task was conducted with a group of right-hemisphere-damaged (RHD) patients and a group of nonbrain-damaged control (NC) participants under three attention conditions-isolation, focused attention, and divided attention-to address the hypothesis that individuals with RHD experience difficulty in the use of contextual information under conditions that tax processing resources. Following Leonard et al. [Leonard, C. L., Baum, S. R., \& Pell, M. D. (2001). The effect of compressed speech on the ability of right-hemisphere-damaged patients to use context. Cortex 37, 327-344], monitoring targets were embedded in three types of sentence contexts: normal, semantically anomalous, and random word order. Results revealed that, under all three attention conditions, monitoring latencies for the RHD patients paralleled those of the NC participants, revealing sensitivity to contextual manipulations. These findings support those of Leonard et al. and suggest that individuals with RHD are, indeed, able to use certain types of contextual information in language processing even under conditions of reduced processing
\end{abstract}

\footnotetext{
* Corresponding author. Present address: School of Rehabilitation Sciences, Faculty of Health Sciences, University of Ottawa, 451 Smyth Road, Ottawa, Ont., Canada K1H 8M5. Tel.: + 1613 562 5800x8300; fax: +1 6135625428 .

E-mail addresses: carol.leonard@uottawa.ca (C.L. Leonard), shari.baum@mcgill.ca (S.R. Baum).
} 
resources. The results are discussed in relation to potential processing distinctions between structural and nonstructural contexts.

(C) 2005 Elsevier Ltd. All rights reserved.

Keywords: Right hemisphere damage; Context use; Word-monitoring; Dual tasks

\section{Introduction}

It has been proposed that the discourse-level deficits found to be characteristic of many individuals with right-hemisphere-damage (RHD) (see Brownell, Gardner, Prather, \& Martino, 1995 for a review), such as difficulty in comprehending narratives (e.g. Hough, 1990; Schneiderman, Murasugi, \& Saddy, 1992), understanding humour (e.g. Shammi \& Stuss, 1999) and understanding nonliteral forms of language (e.g. Kaplan, Brownell, Jacobs, \& Gardner, 1990) may be related to difficulty in the use of contextual information to process language (e.g. Cook, 1989; Cook \& Beech, 1990). This somewhat vague notion of context has not been well defined as pertaining to this claim. Context can embody a number of elements such as the physical surroundings, speaker intentions and relationships during a communicative exchange, the integration of multiple sources of information across a number of sentences, and even structural information. Specifically, with respect to the latter, Fraunfelder and Tyler (1987) have proposed that linguistic contexts may be of two kinds-(1) structural, wherein the processing of linguistic elements contribute to the construction of a higher linguistic unit such as a sentence; and (2) nonstructural, wherein meaning associations between words are developed as they are processed, but no formal linguistic unit is constructed.

Our previous investigations (Leonard \& Baum, 1998; Leonard, Baum, \& Pell, 2001; Leonard, Waters, \& Caplan, 1997a,b) have attempted to systematically investigate the processing of well-defined contexts in order to avoid the potential confounds present in using higher discourse-level tasks. We have argued that it cannot simply be assumed that because success on a particular task requires a sensitivity to contextual considerations (e.g. the interpretation of indirect speech acts), failure on the task necessarily indicates difficulty in using contextual information. Our previous work has, therefore, attempted to qualify the nature of a potential deficit in context use by RHD individuals by clearly manipulating contextual demands. In a series of investigations aimed specifically at targeting the processing of a simple linguistic context, we have consistently found evidence for the ability of individuals with RHD to use context. For example, in Leonard et al. (1997a,b), RHD patients were found to retain the ability to use context to resolve ambiguous pronouns in one- and two-sentence passages. Exploring the possibility that this finding emerged because the task used in those studies, while not on-line, nevertheless tapped automatic processing [and that RHD individuals are able to use context under conditions of reduced processing resources, as advanced by Tompkins and colleagues (e.g. Tompkins, Bloise, Timko, \& Baumgaertner, 1994; Tompkins, Boada, \& McGarry, 1992)], Leonard and Baum (1998) specifically tested the ability of individuals with RHD to use contextual information during automatic on-line processing. Using a word-monitoring task, Leonard and Baum (1998) reported that individuals with RHD were similar to normals in using semantic context, 
as evidenced by faster reaction times to detect a target word in normal sentences (e.g. Tim was experiencing a considerable amount of trouble walking home. The terrible pain was getting increasingly worse in his foot) than in semantically anomalous sentences (e.g. Tim was kissing a proper wall in treatment showing today. The remarkable hotel was doing increasingly wild to his foot). This finding was consistent with previous studies with both young (Marslen-Wilson \& Tyler, 1980) and elderly (Waldstein \& Baum, 1992) normal individuals and it was argued that since both types of sentences were syntactically sound, the facilitation in processing normal versus anomalous sentences must be due to the use of semantic context under the normal context condition.

A critical corollary to the claim that individuals with RHD can use context to process language when the demands of the task do not exceed available processing resources (as in a word-monitoring task) is that, as processing demands increase, a deficit in context use by RHD patients will emerge. This reasoning motivated an investigation by Leonard et al. (2001), in which subjects were required to monitor for words in auditorily presented sentences of three context types-normal, semantically anomalous (as in Leonard and Baum (1998)), and random (both semantically and syntactically anomalous [e.g. In getting the worse terrible increasingly pain his was foot]. In order to evaluate the effect of increased processing demands during performance on the task, the stimuli were presented at three rates of speech-normal, $70 \%$ compressed and $60 \%$ compressed. The results indicated normal effects of semantics and syntax (faster reaction times to anomalous versus random sentences) for both the RHD and normal control groups under the normal rate of speech condition. Using compressed rates of speech, the effect of syntax disappeared for both groups, but the effect of semantics remained. Importantly, the RHD group continued to demonstrate an effect of semantic context under these particular conditions of increased processing demands.

These results were contrary to expectations and two possibilities were advanced to account for the findings. Firstly, the results were discussed relative to the notion of structural and nonstructural contexts. It was proposed that the RHD group was able to use the contextual information in this task because the stimuli under the normal context condition represented a structural context, with the target words completing integrated syntactic/semantic units. This explanation was in keeping with the view, based on data from normal subjects, that the integrity of the $\mathrm{LH}$ is important for the use of syntactic/message level constraints (Faust, 1998; Faust, Babkoff, \& Kravetz, 1995; Faust \& Kravetz, 1998). It was argued that since the LH of the RHD individuals was presumably intact, they were able to use the integrated syntactic/semantic context found under the normal context condition to facilitate processing.

An alternative explanation advanced for the unexpected findings was simply that the demands imposed by the compressed speech conditions were still within the processing capacity of the individuals with RHD who were tested. The present investigation thus sought to re-examine the ability of RHD individuals to use context to monitor for target words under different conditions of increased processing demands, by using a dual task paradigm. A dual task involves the simultaneous performance of an experimental task and an unrelated secondary task. In this case, the experimental task was the word-monitoring task used in Leonard et al. (2001) and the secondary task was an auditory noise/tone discrimination task, modeled after Murray, Holland, and Beeson (1997). Dual tasks are believed to be effortful and 
to increase processing demands in that both tasks are presumably competing for limited shared processing resources (Bower \& Clapper, 1989).

The predictions remained the same as those of Leonard et al. (2001); that is, if RHD selectively impairs context use under effortful conditions, then an effect of semantic context (i.e. faster reaction times to target words in normal than anomalous sentences), expected to hold for both normal and RHD individuals when performing the wordmonitoring task in isolation, should be absent under the dual task condition. Moreover, if RHD selectively impairs semantic context use then an effect of syntax (i.e. faster reaction times to target words in anomalous than random sentences) will continue to hold for both groups under the dual task condition.

\section{Materials and methods}

\subsection{Subjects}

Two groups of subjects participated in this experiment: a group of individuals with righthemisphere damage (RHD) and a group of age- and education-matched nonbrain-damaged normal controls (NC). All subjects were right-handed native speakers of English with hearing within normal limits in at least one ear as determined by a hearing screening at less than $35 \mathrm{~dB}$ HL at the speech frequencies $0.5,1$ and $2 \mathrm{kHz}$. All subjects were also screened on an auditory discrimination task to ensure that they could discriminate between a tone of $1000 \mathrm{~Hz}$ and white noise (a requirement for the experimental task described below).

The RHD group consisted of 12 individuals (mean age: 68.0 years, mean level of education: 15.1 years) who had suffered a single right cerebrovascular accident ${ }^{1}$. Exclusionary criteria included a history of drug or alcohol abuse, and a history of psychiatric or neurological illness. All patients were at least 3 months post-onset at the time of testing and none were aphasic, as determined by available reports. Screening of word and sentence comprehension using subtests from the Psycholinguistic Assessment of Language (Caplan, 1992), however, revealed mild difficulty with the written word-picture matching task for RHD 5 and a mild-moderate deficit in the auditory sentence-picture matching task for RHD subjects 4, 5, and 11. For RHD subjects 5 and 11 this may have been related to attentional problems as noted below. All individuals with RHD were also administered a test battery adapted from the Test of Language Competence-Expanded Edition (Wiig \& Secord, 1987) to evaluate their competence in the areas of comprehension of figurative language and generation of inferences (areas frequently impaired in individuals with RHD). Five of the individuals were identified as having an impairment in generating inferences and ten in comprehending figurative language. Only one subject (RHD 10) did not have any obvious impairment in either domain. Visual neglect was also assessed using the Bells Test (Gauthier, DeHaut, \& Joanette, 1989). One subject (RHD 12) was identified as presenting with visual neglect and three (RHD 2, 5, 11) with possible attentional deficits. Working memory (Tompkins et al., 1994), word span and digit span were also assessed.

\footnotetext{
${ }^{1}$ One participant, RHD8, had some bilateral subcortical involvement.
} 
Table 1

Subject information

\begin{tabular}{|c|c|c|c|c|}
\hline Subject & $\begin{array}{l}\text { Age } \\
\text { (years) }\end{array}$ & $\begin{array}{l}\text { Education } \\
\text { (years) }\end{array}$ & Gender & $\begin{array}{l}\text { Lesion site (acc. to CT scan and/or } \\
\text { neurological report) }\end{array}$ \\
\hline \multicolumn{5}{|l|}{ RHD } \\
\hline 1 & 82 & 15 & $\mathrm{~F}$ & Right internal capsule \\
\hline 2 & 70 & 13 & $\mathrm{~F}$ & Right middle cerebral artery \\
\hline 3 & 63 & 11 & $\mathrm{~F}$ & Right middle cerebral artery \\
\hline 4 & 65 & 12 & M & Not available (NA) \\
\hline 5 & 62 & 14 & M & Right middle cerebral artery \\
\hline 6 & 69 & 20 & M & Right capsulothalamic lacunar \\
\hline 7 & 83 & 17 & M & Right thalamic \\
\hline 8 & 80 & 20 & $\mathrm{~F}$ & Right frontal \\
\hline 9 & 69 & 10 & $\mathrm{~F}$ & NA \\
\hline 10 & 55 & 15 & M & Right parietal \\
\hline 11 & 47 & 16 & $\mathrm{~F}$ & NA \\
\hline 12 & 71 & 18 & M & NA \\
\hline Mean & 68.0 & 15.1 & & \\
\hline \multicolumn{5}{|l|}{$\mathrm{NC}$} \\
\hline 1 & 56 & 17 & $\mathrm{~F}$ & \\
\hline 2 & 77 & 13 & M & \\
\hline 3 & 77 & 14 & $\mathrm{~F}$ & \\
\hline 4 & 58 & 20 & $\mathrm{~F}$ & \\
\hline 5 & 79 & 13 & $\mathrm{~F}$ & \\
\hline 6 & 64 & 15 & $\mathrm{~F}$ & \\
\hline 7 & 65 & 15 & $\mathrm{~F}$ & \\
\hline 8 & 83 & 13 & $\mathrm{~F}$ & \\
\hline 9 & 75 & 9 & M & \\
\hline 10 & 54 & 18 & $\mathrm{~F}$ & \\
\hline Mean & 68.8 & 14.7 & & \\
\hline
\end{tabular}

The NC group consisted of 10 individuals (mean age: 68.8 years; mean level of education: 14.7 years). Exclusionary criteria included a history of drug or alcohol abuse and a history of psychiatric or neurological illness. All subjects underwent a screening to rule out the possibility of dementia and to assess memory skills (see Tables 1 and 2 for subject information).

The groups did not differ significantly in terms of age or education, or on their performance on the word and digit span tasks or the true/false test of the working memory task (Tompkins et al., 1994). They did, however, significantly differ in their performance on the word recall test of the working memory task $[t(20)=4.0, p<0.001$ (2-tailed) (mean \% accuracy 80 versus 65 for the NC and RHD groups, respectively) and their working memory spans $[t(20)=3.05, p<0.01$ (2-tailed)] (3.6 versus 2.5 for the NC and RHD groups, respectively).

\subsection{Stimuli}

The stimuli were those used in Leonard et al. (2001) and consisted of individual sentences, 10 words each in length. The sentences were constructed by manipulating two 
Table 2

Performance on memory tests

\begin{tabular}{|c|c|c|c|c|c|}
\hline & \multicolumn{5}{|c|}{ Working memory (WM) } \\
\hline & True/False (/42) & Word recall (/42) & WM span & Word span & Digit span \\
\hline \multicolumn{6}{|l|}{ RHD } \\
\hline 1 & 42 & 24 & 2 & 4 & 6 \\
\hline 2 & 41 & 28 & 2.5 & 5 & 7 \\
\hline 3 & 41 & 32 & 2.5 & 5 & 6 \\
\hline 4 & 41 & 23 & 2.5 & 4 & 7 \\
\hline 5 & 41 & 25 & 2.5 & 5 & 6 \\
\hline 6 & 42 & 34 & 3 & 5 & 7 \\
\hline 7 & 42 & 22 & 1 & 4 & 8 \\
\hline 8 & 42 & 33 & 3 & 6 & 9 \\
\hline 9 & 42 & 28 & 3.5 & 4 & 8 \\
\hline 10 & 41 & 26 & 2.5 & 4 & 6 \\
\hline 11 & 42 & 28 & 3 & 4 & 6 \\
\hline 12 & 42 & 27 & 2.5 & 4 & 6 \\
\hline Mean & 41.6 & 27.5 & 2.5 & 4.5 & 6.8 \\
\hline \multicolumn{6}{|l|}{$\mathrm{NC}$} \\
\hline 1 & 42 & 36 & 4.5 & 5 & 7 \\
\hline 2 & 42 & 36 & 4 & 6 & 8 \\
\hline 3 & 42 & 35 & 4.5 & 5 & 8 \\
\hline 4 & 42 & 33 & 3.5 & 5 & 7 \\
\hline 5 & 42 & 30 & 3 & 4 & 7 \\
\hline 6 & 42 & 38 & 5 & 4 & 7 \\
\hline 7 & 42 & 33 & 3 & 5 & 8 \\
\hline 8 & 41 & 32 & 3 & 4 & 8 \\
\hline 9 & 42 & 32 & 3 & 4 & 6 \\
\hline 10 & 42 & 29 & 2 & 4 & 7 \\
\hline Mean & 41.9 & $33.4^{\mathrm{a}}$ & $3.6^{\mathrm{a}}$ & 4.6 & 7.3 \\
\hline
\end{tabular}

${ }^{\text {a }}$ Significantly different than the RHD group ( $p<0.01$; two-tailed).

factors-position and context. The factor position had three levels and indicated the position of the target word in the sentence-beginning (3rd or 4th word), middle (5th-7th word), or end (8th-10th word). The factor context also had three levels-normal, anomalous, and random - and was related to the semantic and syntactic integrity of the sentence. Normal sentences were those that were both semantically and syntactically intact (e.g. Jane showed the couple the most charming house in town). Anomalous sentences were semantically anomalous (i.e. did not make sense), but syntactically legal (e.g. Jane left the degree the most meaningful house of land). Anomalous sentences were constructed by changing the content words of the normal sentences (except for the target word), respecting word class and word frequency as much as possible. Random sentences were neither semantically nor syntactically intact (e.g. Couple town the showed charming the Jane house most in) and were constructed by scrambling the words in the normal sentences.

Target words were all monosyllabic with word frequency approximately equally balanced across positions (mean frequencies of 269, 236, and 222 for beginning, middle 
and end positions, respectively [Francis \& Kucera, 1982]). There were 10 different target words at each position, resulting in a total of 90 stimuli.

An adult male native speaker of English recorded the stimuli, making every effort to maintain normal prosody across context types. The stimuli were then digitized using Sound Blaster ${ }^{\circledR} 16$ WavEffects software. The target word was heard first, followed $500 \mathrm{~ms}$ later by the stimulus sentence. The inter-trial interval was $5000 \mathrm{~ms}$. Reaction times (in ms) from the beginning of the target word's appearance in the sentence until a response was made were measured and recorded by the computer.

\subsection{Procedure}

As in Leonard et al. (2001), the stimuli were divided into three blocks of 30 sentences each, such that only one instance of each target appeared within each block. Order of presentation of the stimuli within each block was randomized with the proviso that no more than two consecutive sentences were of the same context type. Once the order of presentation was established in this manner, this fixed order was presented to all subjects. Nine practice trials preceded the presentation of the experimental stimuli.

Similar to Murray et al. (1997), the stimuli were presented in three attention conditions-isolation, focused attention (FA), and divided attention (DA). In the isolation condition, the stimuli were presented without distraction. In both the FA and DA conditions, either a tone of $1000 \mathrm{~Hz}$ or white noise (15 instances of each within a block) was superimposed upon the stimuli. The noise/tone and sentence stimuli were of equal duration and the sentence could be clearly heard over the distracting signal.

Under all conditions, the stimuli were presented to subjects over headphones. Subjects were seated in front of a response box and instructed that they would be hearing a target word followed by a sentence and they were to press the response button as soon as they heard the target word in the sentence. Subjects were encouraged to rest their finger from their unimpaired hand on the response button between trials. The target word was also presented to the subjects visually on a card to reduce memory demands. ${ }^{2}$ Subjects were alerted to the fact that some of the sentences would not make sense.

Under the FA condition, subjects were instructed to listen to the tone/noise, but to only respond when the target word was heard. In the DA condition, subjects were instructed to first respond to the target word with a button press, and then to indicate with a different button press whether a tone or noise was heard on top of the sentence. Subjects were encouraged to guess, if necessary. The order of presentation of the conditions was fixed to be isolation, FA and then DA as recommended by Murray et al. (1997). They argued that the order represents a task hierarchy and it is best to present the less complex conditions first in order to avoid possible feelings of fatigue and failure. Testing in each of the three conditions was separated by at least 1 week.

\footnotetext{
${ }^{2}$ For RHD subjects 7, 8, 9, 10, and 11 cards were not used due to an oversight. In consideration of the finding that no group differences emerged in the analyses, it is believed that this oversight did not affect performance.
} 


\section{Results}

A $2 \times 3 \times 3($ group $\times$ position $\times$ context $)$ analysis of variance with both subjects $(\mathrm{F} 1)$ and items (F2) as random factors was performed on the mean reaction times (RTs), separately for each attention condition. Reaction times greater than $5 \mathrm{~s}$ were automatically timed out and not included in the analysis. As well, extreme reaction time values defined as those greater than two standard deviations from the mean, calculated per subject, per sentence condition, were also not included in the analysis. This resulted in the exclusion of 4.6 and $6.1 \%$ of responses for the $\mathrm{NC}$ and RHD groups, respectively, under the isolation condition; $6.7 \%$ and $12.2 \%$ of responses for the NC and RHD groups, respectively, under the FA condition; and 6.4 and $6.1 \%$ of responses for the NC and RHD groups, respectively, under the DA condition. Only significant effects that either passed Mauchly's test of sphericity for homogeneity of covariance or if, having failed $(p<0.05)$, remained significant using the more conservative Greenhouse-Geisser test are reported. Individual patterns of performance are also reported as meaningful if a difference of at least $20 \mathrm{~ms}$ was found between the conditions of interest.

Fig. 1 shows the mean RTs to target words by position and context for each group under the isolation condition. The analysis revealed significant main effects of position [F1 (2, $40)=11.3, \mathrm{MSE}=16,999.2, p<0.01 ; \mathrm{F} 2(2,81)=20.0, \mathrm{MSE}=9036.2, p<0.001]$ and context $[\mathrm{F} 1(2,40)=95.6, \mathrm{MSE}=2004.2, p<0.001 ; \mathrm{F} 2(2,81)=17.9, \mathrm{MSE}=9036.2, p<$ $0.001)]$. The main effect of group was significant by items only $[\mathrm{F} 1(1,20)=2.4$, MSE $=$ $109,501.1 ; \mathrm{F} 2(1,81)=130.0, \mathrm{MSE}=1900.3, p<0.001]$. Post hoc analyses using the Newman-Keuls procedure $(p<0.05)$ revealed that RTs to target words in the beginning position were slower (mean $=470 \mathrm{~ms}$ ) than to those in the middle and end positions
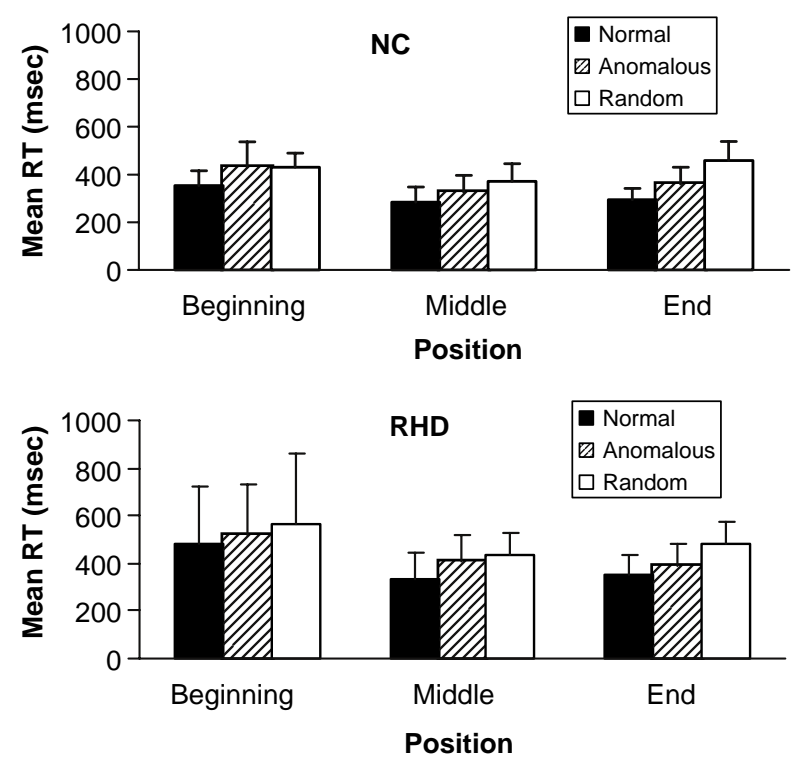

Fig. 1. Mean reaction times (ms) to target words by position and context under the isolation condition. 

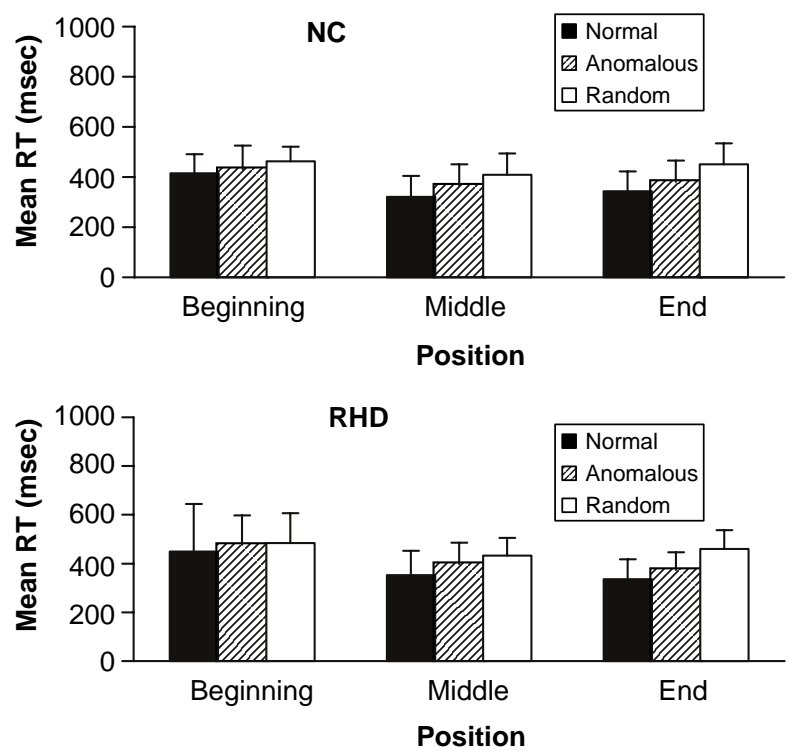

Fig. 2. Mean reaction times (ms) to target words by position and context under the focused attention condition.

(means $=363 \mathrm{~ms}$ and $391 \mathrm{~ms}$, respectively), which did not significantly differ from each other. RTs were also faster to target words under the normal context (mean $=354 \mathrm{~ms}$ ) than the anomalous context (mean $=409 \mathrm{~ms}$ ), which was, in turn, faster than the random (mean $=462 \mathrm{~ms})$ context.

Inspection of the individual data revealed that the effect of position was relatively robust with $60 \%$ of the NC group and $67 \%$ of the RHD group evidencing slower RTs to targets in the beginning than end positions and $100 \%$ of both groups evidencing slower RTs to targets in the beginning than middle positions. The effect of context was quite robust with 80 and $83 \%$ of the NC and RHD groups, respectively, demonstrating an effect of semantic context, reflected in faster RTs to targets in normal versus anomalous sentences, and 90 and $92 \%$ of the NC and RHD groups, respectively, demonstrating an effect of syntactic context (i.e. faster RTs to targets in anomalous versus random sentences).

The interaction position $\times$ context was also significant, but by subjects only $[\mathrm{F} 1(4,80)=$ 5.3, $\mathrm{MSE}=1881.2, p<0.01 ; \mathrm{F} 2(4,81)=0.96, \mathrm{MSE}=9036.2]$. An analysis of simple main effects, using the Bonferroni adjustment for multiple comparisons, revealed that while both effects of semantic and syntactic context held at the middle and end positions, only the effect of semantic context held at the beginning position. No other interactions were significant by subject. The interaction group $\times$ position was significant by items only $[F 1(2,40)=1.7$, $\mathrm{MSE}=16,999.2 ; \mathrm{F} 2(2,81)=12.8, \mathrm{MSE}=1900.3, p<0.001]$

Fig. 2 shows the mean RTs to target words by position and context for each group under the FA condition ${ }^{3}$. The analysis revealed significant main effects of position

\footnotetext{
${ }^{3}$ RHD12 was not tested under this or the DA condition.
} 
$[\mathrm{F} 1(2,38)=16.3, \mathrm{MSE}=6035.0, p<0.001 ; \mathrm{F} 2(2,81)=13.0, \mathrm{MSE}=8544.6, p<0.001]$ and context $[\mathrm{F} 1(2,38)=36.9, \mathrm{MSE}=2774.9, p<0.001 ; \mathrm{F} 2(2,81)=12.2, \mathrm{MSE}=8544.6$, $p<0.001)]$. The main effect of group was significant by items only $[\mathrm{F} 1(1,19)=0.31$, $\mathrm{MSE}=62,686.1 ; \mathrm{F} 2(1,81)=11.3, \mathrm{MSE}=1452.4, p<0.01]$. Post hoc analyses using the Newman-Keuls procedure $(p<0.05)$ once again revealed that RTs to target words in the beginning position were slower $($ mean $=454 \mathrm{~ms}$ ) than to those in the middle and end positions (means $=380 \mathrm{~ms}$ and $391 \mathrm{~ms}$, respectively), which did not significantly differ from each other. RTs were also faster to target words under the normal context (mean= $370 \mathrm{~ms}$ ) than both the anomalous (mean $=405 \mathrm{~ms}$ ) and random (mean $=450 \mathrm{~ms}$ ) contexts, and faster under the anomalous than the random context.

Inspection of the individual data revealed that the effect of position was robust with $100 \%$ of the NC group and $91 \%$ of the RHD group evidencing slower RTs to targets in the beginning than the middle and $70 \%$ or the NC group and $82 \%$ of the RHD group demonstrating slower RTs to targets in the beginning than end positions. The effect of context was also strong with 70 and $73 \%$ of the NC and RHD groups, respectively, demonstrating an effect of semantic context, reflected in faster RTs to targets in normal versus anomalous sentences, and 70 and $73 \%$ of the NC and RHD groups, respectively, demonstrating an effect of syntactic context (i.e. faster RTs to targets in anomalous versus random sentences).

The interaction position $\times$ context was also significant, but by subjects only [F1 (4, $76)=6.1, \mathrm{MSE}=1369.9, p<0.01 ; \mathrm{F} 2(4,81)=1.0, \mathrm{MSE}=8544.6]$. An analysis of simple main effects, using the Bonferroni adjustment for multiple comparisons, revealed that while both effects of semantic and syntactic context held at the middle and end positions, neither effect held at the beginning position. No other interactions were significant by subject. The interaction group $\times$ position was significant by items only $[F 1(2,38)=0.95$, $\mathrm{MSE}=6035.0 ; \mathrm{F} 2(2,81)=6.0, \mathrm{MSE}=1452.4, p<0.01]$.

Fig. 3 shows the mean RTs to target words by position and context for each group under the DA condition ${ }^{4}$. The analysis revealed significant main effects of position $[\mathrm{F} 1(2,36)=$ 35.1, $\mathrm{MSE}=15,705.3, p<0.001 ; \mathrm{F} 2(2,81)=41.5, \mathrm{MSE}=14,233.7, p<0.001]$ and context $[\mathrm{F} 1(2,36)=7.1, \mathrm{MSE}=7540.7, p<0.01 ; \mathrm{F} 2(2,81)=3.3, \mathrm{MSE}=14,223.7, p<$ $0.05)]$. The effect of group was significant by items only $[\mathrm{F} 1(1,18)=0.18$, MSE $=$ $317,280.5 ; \mathrm{F} 2(1,81)=12.65, \mathrm{MSE}=3889.5, p<0.01]$. No interactions were significant by subjects or items. Post hoc analyses using the Newman-Keuls procedure $(p<0.05)$ revealed that RTs to target words in the beginning position were slower (mean $=688 \mathrm{~ms}$ ) than to those in the middle and end positions (means $=536$ and $511 \mathrm{~ms}$, respectively), which did not significantly differ from each other. RTs were also faster to target words under the normal context (mean $=547 \mathrm{~ms}$ ) than both the anomalous (mean $=581 \mathrm{~ms}$ ) and random (mean $=607 \mathrm{~ms}$ ) contexts, which were not significantly different from each other.

Inspection of the individual data revealed that the effect of position was once again quite robust. The finding of slower RTs to targets at the beginning of sentences compared to both the middle and end positions was found for $90 \%$ of subjects in the NC group

\footnotetext{
${ }^{4}$ It was noted that for RHD5, for 4 of the 9 sentence conditions, more than half of the RTs were 0. As a result, this subject's data were not included in the analysis.
} 

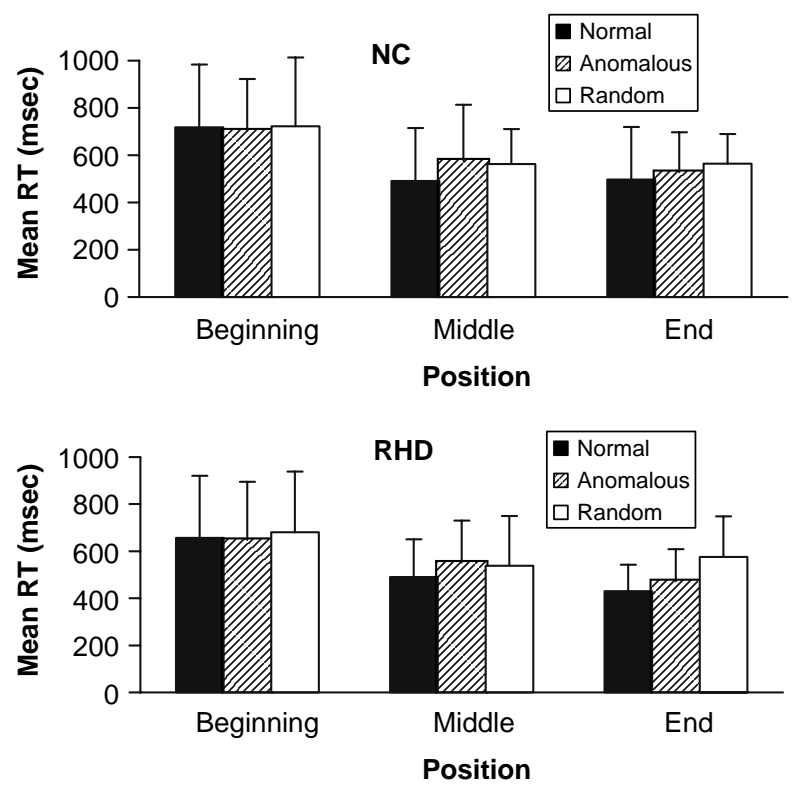

Fig. 3. Mean reaction times (ms) to target words by position and context under the divided attention condition.

and $100 \%$ of subjects in the RHD group. The effect of semantic context (normal faster than anomalous) was less robust than in the either the isolation or FA conditions, demonstrated by only $60 \%$ of both the NC and RHD groups. No interactions were significant ${ }^{5}$.

Performance on the discrimination task was extremely accurate for both groups (mean \% errors: 1.32 versus 3.22 for the NC and RHD groups, respectively) and was not significantly different between groups.

\section{Discussion}

The purpose of this experiment was to explore the possibility that a failure to find a deficit in context use by individuals with RHD in Leonard et al. (2001) was related to the fact that compressed rates of speech did not sufficiently burden the system such that the task was beyond the processing capabilities of the RHD individuals tested. Thus, a different paradigm, using a dual-task procedure, was employed in an attempt to place a higher cognitive demand on the participants while engaging in the word-monitoring task. The dual task paradigm has been used successfully in other work investigating attention and resource allocation deficits in aphasia (e.g. Murray et al., 1997).

The results were conclusive in supporting those of Leonard et al. (2001) that individuals with RHD can use context to process language even under conditions of increased

\footnotetext{
${ }^{5}$ A significant position $\times$ context $\times$ group interaction, based on an analysis of the incomplete data set, was incorrectly reported in Leonard and Pell (2001) due to a coding error during the analysis.
} 
processing demands. The effect of semantic context was extremely robust and evidenced by both the NC and RHD groups under all three attention conditions. Interestingly, despite the variability of lesion site in the RHD group, an inspection of individual patterns of performance found the effect of semantic context to be quite uniform across the majority of individuals in the RHD group and comparable to the individuals in the NC group. This latter observation suggests that a failure to find a deficit in context use by the RHD group is not likely related to lesion site. Furthermore, while it is true that under the DA condition the effect of semantic context was somewhat weaker, in that only $60 \%$ of both the NC and RHD groups demonstrated the effect, the important point to note is that the DA condition did not differentially affect the ability of individuals with RHD to use context. In fact, this finding probably attests more to the fact that, indeed, the DA condition did impose a higher cognitive demand on all participants, refuting also the possibility that learning may have occurred across the testing sequence, potentially obviating group differences. Further support for the notion that the DA condition was, indeed, a more difficult condition, is found in the finding that, while the effect of syntax was robust under both the isolation and FA conditions, it failed to emerge under the DA condition. In fact, this pattern of results is an exact replication of the results of Leonard et al. (2001) in which it was found that the independent facilitory effect of syntax, found under the normal rate of speech condition, disappeared under both compressed rate of speech conditions.

It seems that the most plausible explanation for the results found in the present investigation and those in Leonard et al. (2001) relates to the distinction, as already noted in Leonard et al. (2001), between structural and nonstructural contexts. It has been found in studies with normals that the $\mathrm{RH}$ is important in the processing of nonstructural contexts - in the activation and maintenance of peripheral meaning associations between words (Beeman, 1993, 1998; Faust \& Kahana, 2002; Faust \& Lavidor, 2003; Titone, 1998). To the extent that the stimuli used in the present study represented a structural context, it is perhaps not surprising that the individuals with RHD were able to use their intact $\mathrm{LH}$ to aid in the processing of the sentences and the detection of the target words.

It is interesting to note that the results of the present study are consistent with the findings of a recent study by Faust, Bar-Lev, and Chiarello (2003) investigating the contributions of syntactic and semantic information to sentence priming in the two cerebral hemispheres. Using a divided visual field paradigm, young normal subjects were required to make a lexical decision to a target word presented to either the right visual field $(\mathrm{LH})$ or left visual field $(\mathrm{RH})$ that completed a centrally presented priming sentence. The priming sentences were of five types, three of which corresponded to the context types used in the present study; that is, normal, semantically anomalous and random. The target words were semantically associated to single words in the sentences. The results indicated priming in both hemispheres for the normal sentence primes and moderate priming in both hemispheres for the random sentence primes. Interestingly, there was no priming in the LH for the semantically anomalous sentence primes, but there was in the RH. The authors interpreted this finding to suggest that once the LH engages in syntactic parsing, the activation of the meanings of words will only be maintained if the meanings are coherent. In contrast, the RH does not require coherence for the maintenance of multiple word meanings. This view, consistent with the role of the $\mathrm{RH}$ as a processor of nonstructural contexts, is also compatible with the results of the present study in which 
a word-monitoring task was used. For the RHD patients in the present study, the results revealed an advantage in processing when both semantic and syntactic information were available (as in the normal condition), and also when only syntactic information was present (as in the semantically anomalous condition), permitting listeners to anticipate the presence of a noun. Assuming that the intact LH in these individuals provided the syntactic analysis, while the individual word meanings may not have been maintained by the LH due to lack of coherence (according to Faust et al.'s (2003) hypothesis), the maintenance of word meanings is not required for an effect to emerge in the word-monitoring task because it does not require activation of semantic associates. In Faust et al. (2003), on the other hand, the task was that of lexical decision, in which target words were semantically associated to single words in the sentence. In order to obtain priming in such a task, the meanings invoked by the words had to be maintained.

The foregoing discussion underscores an important point in research investigating the laterality of context use in language processing. Not only must the type of context (structural versus nonstructural) be considered, but also the processing (i.e. syntactic, semantic, inferential, etc.) invoked by the particular task used, as well as its processing resource demands (Tompkins et al., 1994). Related to the latter is the notion of working memory. Tompkins et al. (1994) found a relationship between working memory capacity and discourse comprehension. Interestingly, with regard the present study, while the NC and RHD groups differed significantly in measures of working memory, but not short-term memory, no group differences emerged on the experimental tasks suggesting that the word-monitoring task, even under the demanding dual task condition, did not tax working memory processes.

In conclusion, the results of the present investigation are consistent with our previous studies which have carefully manipulated levels of context use and processing demands (Leonard \& Baum, 1998; Leonard et al., 2001, 1997a,b), and suggest that the claim that the $\mathrm{RH}$ is specialized for the processing of contextual information and therefore that RHD individuals have difficulty using context to process language (e.g. Cook \& Beech, 1990) is a far too simplistic and ill-defined notion. Factors such as type of context and demands related to manipulation of multiple sources of context and meaning such as working memory must be considered in order to better elucidate the role of the right hemisphere in language processing.

\section{Acknowledgements}

Portions of this work were presented at the Academy of Aphasia meeting, Boulder, Colorado, October, 2001 (Leonard \& Pell, 2001). This is an Isabel Silverman Canada International Scientific Exchange Program project, supported by the Saul A. Silverman Family Foundation, Toronto, Canada, and a grant to the first author from the Natural Sciences and Engineering Research Council of Canada. The authors are grateful to the Toronto Rehabilitation Institute for allowing us to recruit subjects from their institution and to all the individuals who participated in this research. 


\section{References}

Beeman, M. (1993). Semantic processing in the right hemisphere may contribute to drawing inferences from discourse. Brain and Language, 44, 80-120.

Beeman, M. (1998). Coarse semantic coding and discourse comprehension. In M. Beeman, \& C. Chiarello (Eds.), Right hemisphere language comprehension: Perspectives from cognitive neuroscience (pp. 255-284). Mahwah, NJ: Lawrence Erlbaum Associates.

Bower, G. H., \& Clapper, J. P. (1989). Experimental methods in cognitive science. In M. I. Posner (Ed.), Foundations of cognitive science (pp. 261-262). Cambridge: MIT Press, 261-262.

Brownell, H., Gardner, H., Prather, P., \& Martino, G. (1995). Language, communication, and the right hemisphere. In H. S. Kirshner (Ed.), Handbook of neurological speech and language disorders (pp. 325-349). New York: Marcel Dekker, 325-349.

Caplan, D. (1992). Language: Structure, process and disorders. Cambridge, MA: MIT Press.

Cook, N. D. (1989). Toward a central dogma for psychology. New Ideas in Psychology, 7, 1-18.

Cook, N. D., \& Beech, A. R. (1990). The cerebral hemispheres and bilateral neural nets. International Journal of Neuroscience, 52, 201-210.

Faust, M. (1998). Obtaining evidence of language comprehension from sentence priming. In M. Beeman, \& C. Chiarello (Eds.), Right hemisphere language comprehension: Perspectives from cognitive neuroscience (pp. 161-185). Mahwah, NJ: Lawrence Erlbaum Associates.

Faust, M., Babkoff, H., \& Kravetz, S. (1995). Linguistic processes in the two cerebral hemispheres: Implications for modularity vs. interactionism. Journal of Clinical and Experimental Neuropsychology, 17, 171-192.

Faust, M., Bar-Lev, A., \& Chiarello, C. (2003). Sentence priming effects in the two cerebral hemispheres: Influences of lexical relatedness, word order, and sentence anomaly. Neuropsychologia, 41, 480-492.

Faust, M., \& Kahana, A. (2002). Priming summation in the cerebral hemispheres: Evidence from semantically convergent and semantically divergent primes. Neuropsychologia, 40, 892-901.

Faust, M., \& Kravetz, S. (1998). Levels of sentence constraint and lexical decision in the two hemispheres. Brain and Language, 62, 149-162.

Faust, M., \& Lavidor, M. (2003). Semantically convergent and semantically divergent priming in the cerebral hemispheres: Lexical decision and semantic judgment. Cognitive Brain Research, 17, 585-597.

Francis, W. N., \& Kucera, H. (1982). Frequency analysis of English usage. Boston, MA: Houghton Mifflin Co.

Fraunfelder, U. H., \& Tyler, L. K. (1987). The process of spoken word recognition: An introduction. Cognition, $25,1-20$.

Gauthier, L., Dehaut, F., \& Joanette, Y. (1989). The Bells Test: A quantitative and qualitative test for visual neglect. International Journal of Clinical Neuropsychology, 11, 49-54.

Hough, M. S. (1990). Narrative comprehension in adults with right and left hemisphere damage: Theme organization. Brain and Language, 38, 253-277.

Kaplan, J. A., Brownell, H. H., Jacobs, J. R., \& Garnder, H. (1990). The effects of right hemisphere damage on the pragmatic interpretation of conversational remarks. Brain and Language, 38, 315-333.

Leonard, C. L., \& Baum, S. R. (1998). On-line evidence for context use by right brain damaged patients. Journal of Cognitive Neuroscience, 10(4), 499-508.

Leonard, C. L., Baum, S. R., \& Pell, M. D. (2001). The effect of compressed speech on the ability of righthemisphere-damaged patients to use context. Cortex, 37, 327-344.

Leonard, C. L., \& Pell, M. D. (2001). Context use by right-hemisphere-damaged individuals under conditions of focused and divided attention: Preliminary findings. Brain and Language, 79(1), 59-61.

Leonard, C. L., Waters, G. S., \& Caplan, D. (1997a). The use of contextual information by right brain-damaged individuals in the resolution of ambiguous pronouns. Brain and Language, 57, 309-342.

Leonard, C. L., Waters, G. S., \& Caplan, D. (1997b). The use of contextual information related to general world knowledge by right brain-damaged individuals in pronoun resolution. Brain and Language, 57, 343-359.

Marslen-Wilson, W., \& Tyler, L. K. (1980). The temporal structure of spoken language understanding. Cognition, $8,1-71$.

Murray, L. L., Holland, A. L., \& Beeson, P. M. (1997). Auditory processing in individuals with mild aphasia: A study of resource allocation. Journal of Speech, Language, and Hearing Research, 40, 792-808. 
Schneiderman, E. I., Murasugi, K. G., \& Saddy, J. D. (1992). Story-arrangement ability in right brain-damaged patients. Brain and Language, 43, 107-120.

Shammi, P., \& Stuss, D. T. (1999). Humour appreciation: A role of the right frontal lobe. Brain, 122, 657-666.

Titone, D. (1998). Hemispheric differences in context sensitivity during lexical ambiguity resolution. Brain and Language, 65, 361-394.

Tompkins, C. A., Bloise, C. G. R., Timko, M. L., \& Baumgaertner, A. (1994). Working memory and inference revision in brain-damaged and normally aging adults. Journal of Speech and Hearing Research, 37, 896-912.

Tompkins, C. A., Boada, R., \& McGarry, K. (1992). The access and processing of familiar idioms by braindamaged and normally aging adults. Journal of Speech and Hearing Research, 35, 626-637.

Waldstein, R. S., \& Baum, S. R. (1992). The influence of syntactic and semantic context on word-monitoring latencies in normal aging. Journal of Speech Language Pathology and Audiology, 16, 217-222.

Wiig, E. H., \& Secord, W. (1987). Test of language competence - Expanded edition. San Antonio: Harcourt, Brace, and Jovanovich. 